\title{
Efectos de la variación de la intensidad de los tonos primarios sobre las amplitudes de Emisiones Otoacústicas Producto de Distorsión (EOApd) en sujetos con audición dentro de rangos de normalidad
}

\section{Effects of varying the intensity of the primary tones on the amplitudes of Distortion Product Otoacoustic Emissions (OAEdp) in patients with normal hearing}

\author{
Karina Pinochet $\mathbf{R}^{1}$, Karen Alegría $\mathbf{B}^{1}$, Marcelo Romero A1 ${ }^{1}$ Oscar Cañete $\mathbf{S}^{2}$.
}

\begin{abstract}
RESUMEN
Introducción: Las emisiones otoacústicas (EOA) son sonidos generados por las céIulas ciliadas externas (CCE). Se ha visto que la generación y registro de las EOA evocadas depende de factores técnicos de evaluación como el nivel de intensidad de los tonos primarios, relación frecuencial entre los tonos, etc.

Objetivos: Estudiar el efecto de la variación de la intensidad de los tonos primarios en la amplitud de la respuesta de las EOA producto de distorsión ( $p d)$ en individuos con audición normal.

Material y método: Se evaluaron 35 individuos a los cuales se les midieron EOApd utilizando diferentes intensidades de tonos primarios: Intensidad baja $(I 1=50 \mathrm{dBSPL} e$ $12=50 \mathrm{dBSPL})$, intensidad media $(11=55 \mathrm{dBSPL}$ e $12=65 \mathrm{dBSPL})$ e intensidad alta (I1=70dBSPL e I2=70dBSPL).

Resultados: Se observó que para tonos primarios a bajas intensidades ( $11=50 \mathrm{dBSPL}$ e 12=50dBSPL) existe una disminución tanto en amplitud y presencia de las EOApd, mientras que para tonos primarios a intensidades mayores $(11=55 \mathrm{dBSPL}$ e $12=65 \mathrm{dBSPL}$ - I1=70dBSPL e I2=70dBSPL) se evidenció un aumento en amplitud y presencia de EOApd en los sujetos estudiados.

Conclusión: Se evidencian diferencias estadísticamente significativas en la amplitud de las EOApd al variar la intensidad de los tonos primarios. Por lo cual se puede establecer que las intensidades medias-altas son las más adecuadas para la evaluación de EOApd en sujetos con audición normal.

Palabras clave: Emisiones otoacústicas, tonos primarios, intensidad de estimulación, variación de tonos primarios.
\end{abstract}

\section{ABSTRACT}

Introduction: Otoacoustic emissions (OAE) are sounds produced by outer hair cells. The generation and recording of evoked OAE depends on technical factors such as level of intensity of primary tones, frequency relationship between the tones, etc.

1 Escuela de Tecnología Médica, Facultad de Medicina, Viña del Mar, Universidad Andrés Bello.

2 Tecnólogo Médico, Departamento de Audición y Lenguaje, Servicio de Otorrinolaringología, Hospital Padre Hurtado. 
Aim: To study the effect of varying the intensity of primary tones in the amplitude of the response of Distortion Product OAE (DP) in individuals with normal hearing.

Material and method: 35 individuals were measured using different intensities OAEdp primary tones: Low Intensity $(I 1=12=50 \mathrm{dBSPL} 50 \mathrm{dBSPL}$ e), Medium Intensity $(11=12$ $=65 d B S P L$ 55dBSPL e) and High Intensity ( $I 1=70 d B S P L$ and $I 2=70 d B S P L)$.

Results: Using Low primary tones ( $11=12=50 \mathrm{dBSPL} 50 \mathrm{dBSPL}$ ) there is a decrease in amplitude and presence of DPOAE, while for primary tones at higher intensities (11 and $12==55 d B S P L$ 65dBSPL $-11=70 d B S P L$ and $12=70 d B S P L)$ an increase in amplitude and presence of OAEdp in subjects with normal hearing was observed .

Conclusion: There are statistically significant differences in OAEdp amplitude by varying the intensity of the primary tones. Therefore medium-high intensities are best suited for the evaluation of OAEdp in subjects with normal hearing.

Key words: Otoacustic Emissions, primary levels, tones levels, tones variation, distortion product.

\section{INTRODUCCIÓN}

Las EOA corresponden a respuestas sonoras generadas por las células ciliadas externas (CCE) del oído interno en forma espontánea o provocada, pudiendo ser registradas a través de un micrófono ubicado a nivel del conducto auditivo externo (CAE). Un tipo de EOAs provocadas se denominan producto de distorsión ( $p d$ ) las cuales se obtienen en respuesta al presentar dos tonos de diferentes frecuencias ( $f 1$ y f2 0 tonos primarios) en forma simultánea ${ }^{1}$.

Las EOApd corresponden a las distorsiones resultantes del comportamiento no lineal de la cóclea, es una medición simultánea a la presentación de dos tonos puros designados como f1 y f2, en donde siempre $\mathrm{f} 1<\mathrm{f} 2$, con intensidades designadas como L1 y L2, respectivamente. La amplitud de los productos de distorsión varía individualmente, sin embargo, la amplitud típica de producto de distorsión reportada para adultos va en el rango de 45 a $75 \mathrm{~dB}$ bajo el nivel de equivalencia de los tonos primarios. El registro y obtención de EOApd se relaciona con un umbral auditivo mejor 0 igual a 50-55 dB HL junto con indemnidad de oído medio y externo2. Diversos parámetros técnicos son críticos cuando se registran los productos de distorsión, dentro de éstos se encuentran la frecuencia, relación de frecuencia, nivel de estímulo, y diferencia en el nivel de estímulo.

Junto con la relación de frecuencia, es necesaria la diferencia de intensidad de los dos tonos primarios (L1/L2), pues también afecta la amplitud. Las prime- ras investigaciones sobre estos factores utilizaron iguales niveles de presión sonora $(\mathrm{L} 1-\mathrm{L} 2=0)$ de los tonos primarios en medición del 2f1-f2. Manipulando niveles de estímulo de f1 y f2 de forma independiente, se encontró que es necesaria la diferencia de L1 y L2 para producir un nivel robusto de respuesta 2f1-f2 que varía según el nivel de estímulo global'.

Por lo anteriormente expuesto sería de interés establecer el efecto que tiene la variación de la intensidad de tonos primarios sobre la amplitud de las EOApd en sujetos que presentan audición dentro de rangos de normalidad con el fin de determinar cuál es el parámetro de intensidad más adecuado para el estudio de la función coclear en esta población. Esto sería de utilidad ya que a la fecha no existen datos a nivel nacional que evalúen estos parámetros por lo tanto ayudaría al mejoramiento de las técnicas de evaluación de la función coclear como lo son las EOApd.

\section{OBJETIVOS}

El objetivo de este trabajo fue estudiar el efecto de la variación de la intensidad de los tonos primarios en la amplitud de la respuesta de las EOA producto de distorsión (pd) en individuos con audición normal.

\section{MATERIAL Y MÉTODO}

Se evaluaron 35 individuos entre 17 y 30 años de edad estudiantes de la carrera de Tecnología Médica de la 
Universidad Andrés Bello sede Viña del Mar, en el periodo mayo-junio 2011, que cumplieron los siguientes criterios de inclusión: a) Audición dentro de rangos de normalidad en ambos oídos ( $\leq 20 \mathrm{~dB} \mathrm{HL}$ entre 125 y $8000 \mathrm{~Hz}$ ), b) Timpanograma «A», presencia de reflejo acústico en las frecuencias de 500,1.000, 2.000 y $4.000 \mathrm{~Hz}$, (ipsi/ contralateral), c) Sin antecedentes de patología otológica reciente (periodo de 3 meses previos), d) Sin antecedentes de exposición a ototóxicos y de exposición a ruido (permanente y 72 horas previas a la evaluación).

En una primera etapa cada individuo respondió un cuestionario para evaluar conductas auditivas, uso de medicamentos ototóxicos y enfermedades auditivas. Luego se realizó la evaluación auditiva correspondiente y para ello se utilizó un audiómetro Interacoustics (modelo AC-40) e impedanciómetro Interacoustics (modelo AT 235). Cada individuo firmó el consentimiento informado correspondiente.

Aquellos individuos que cumplieron los criterios de inclusión fueron citados a una segunda sesión donde se realizó la medición de EOApd clínicas (Otoport Advance, Otodynamics). Cada sujeto fue evaluado siguiendo un protocolo de medición previamente establecido, el cual contempla sólo la variación en la intensidad y orden de presentación (intensidades media, baja y alta) sin alteración de otros parámetros como tiempo de evaluación, valor de señal/ruido, criterio pasa, relación f2/f1. Estas intensidades fueron seleccionadas ya que son las que se encuentran descritas en la literatura para su uso en investigación y/o uso clínico. Cada individuo evaluado participó en forma voluntaria, obteniéndose su autorización a través del consentimiento informado correspondiente.

Con la finalidad de establecer la normalidad de la muestra se aplicó la prueba de KolmogorovSmirnov ( $p \leq 0,05$, $p$-valor 0,070 ), observando una distribución normal de ésta. Se utilizaron medidas de resumen y análisis de varianza (ANOVA).

\section{RESULTADOS}

Entre mayo y junio de 2011 se evaluaron 35 pacientes, $57,1 \%$ (20) sexo femenino y de $42,9 \%$ (15) sexo masculino. Los valores de la amplitud registrados fueron en respuesta a diferentes intensidades de presentación, frecuencia, género y oído. El análisis individual de los factores permite concluir que no existe diferencia significativa en la amplitud según género y oído. ( $\alpha=5 \%$ ). Sin embargo sí existe diferencia significativa en la amplitud según la intensidad y frecuencia. (Figura 1).

Al utilizar tonos primarios de baja intensidad (50-50 dBSPL) se pudo observar valores promedios de amplitud entre $-16.45 \mathrm{dBSPL}$ y $-0.05 \mathrm{dBSL}$ para la frecuencia de $8.000 \mathrm{~Hz}$ y $1.500 \mathrm{~Hz}$ respectivamente (Tabla 1). Los rangos de amplitud al utilizar tonos primarios de intensidad media (50-65 $\mathrm{dBSPL}$ ) varían desde un -6.06 dBSPL y 13.78 dBSPL para la frecuencia de $8.000 \mathrm{~Hz}$ y $1.500 \mathrm{~Hz}$ respectivamente (Tabla 2). Para intensidades mayores (70-70 dBSPL) las amplitudes varían desde $2.96 \mathrm{dBSPL}$ y $14.85 \mathrm{dBSPL}$ para las frecuencias de $8.000 \mathrm{~Hz}$ y $6.000 \mathrm{~Hz}$ respectivamente (Tabla 3).

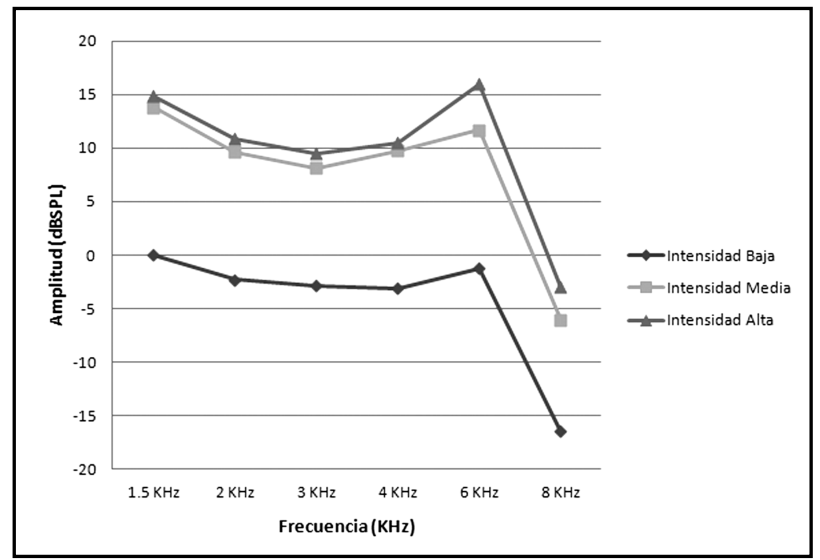

Figura 1. Amplitud promedio (dB SPL) a diferente intensidad de tonos primarios entre las frecuencias de 1,5 kHz y $8 \mathrm{kHz}$. 
Tabla 1. Medidas de resumen para cada frecuencia estudiada a intensidades bajas de tonos primarios (50-50 dBSPL)

\begin{tabular}{|lccccc|}
\hline Frecuencia & OBS. & Promedio & DE & Min & Max \\
\hline $1.500 \mathrm{~Hz}$ & 35 & -.0057143 & 7.150358 & -15.1 & 10.8 \\
$2.000 \mathrm{~Hz}$ & 35 & -2.288571 & 5.922627 & -15.6 & 6.5 \\
$3.000 \mathrm{~Hz}$ & 35 & -2.865714 & 7.183189 & -17.1 & 10.4 \\
$4.000 \mathrm{~Hz}$ & 35 & -3.125714 & 7.831135 & -22.1 & 9.9 \\
$6.000 \mathrm{~Hz}$ & 35 & -1.248571 & 7.441472 & -14.7 & 15.2 \\
$8.000 \mathrm{~Hz}$ & 35 & -16.45429 & 4.876193 & -27 & -6.2 \\
\hline
\end{tabular}

Tabla 2. Medidas de resumen para cada frecuencia estudiada a intensidades medias de tonos primarios (50-65 dBSPL)

\begin{tabular}{|lccccc|}
\hline Frecuencia & OBS. & Promedio & DE & Min & Max \\
\hline $1.500 \mathrm{~Hz}$ & 35 & 13.78857 & 7.225314 & -19.5 & 21.9 \\
$2.000 \mathrm{~Hz}$ & 35 & 9.637143 & 6.570258 & -9 & 22 \\
$3.000 \mathrm{~Hz}$ & 35 & 8.117143 & 5.601706 & -2.8 & 17.6 \\
$4.000 \mathrm{~Hz}$ & 35 & 9.785714 & 7.187127 & -8.2 & 19.4 \\
$6.000 \mathrm{~Hz}$ & 35 & 11.67429 & 5.868277 & -1.6 & 22.5 \\
$8.000 \mathrm{~Hz}$ & 35 & -6.065714 & 6.663639 & -19.9 & 6.4 \\
\hline
\end{tabular}

Tabla 3. Medidas de resumen para cada frecuencia estudiada a intensidades medias de tonos primarios (70-70 dBSPL)

\begin{tabular}{|lccccc|}
\hline Frecuencia & OBS. & Promedio & DE & Min & Max \\
\hline $1.500 \mathrm{~Hz}$ & 35 & 14.85429 & 4.860364 & 0.4 & 22.9 \\
$2.000 \mathrm{~Hz}$ & 35 & 10.85143 & 6.022476 & -3.9 & 21.9 \\
$3.000 \mathrm{~Hz}$ & 35 & 9.505714 & 5.46771 & -1.0 & 19.5 \\
$4.000 \mathrm{~Hz}$ & 35 & 10.53143 & 6.735716 & -3.9 & 20.3 \\
$6.000 \mathrm{~Hz}$ & 35 & 16.02286 & 6.470571 & 3.1 & 26 \\
$8.000 \mathrm{~Hz}$ & 35 & -2.968571 & 8.004218 & -19.0 & 13.2 \\
\hline
\end{tabular}

Se evidencia que al utilizar tonos primarios de baja intensidad $(I 1=50 \mathrm{dBSPL}$ e $\mathrm{I} 2$ $=50 \mathrm{dBSPL}$ ) la presencia de EOApd varía desde $1,5 \%(8.000 \mathrm{~Hz})$ hasta $55,8 \%(2.000 \mathrm{~Hz})$ en el total de los oídos estudiados (Tabla 4). Siendo la frecuencia de $8.000 \mathrm{~Hz}$ a su vez la con menor amplitud de EOApd al analizar las 3 intensidades estudiadas (Figura 1). Por otro lado a intensidades altas $(11=70 \mathrm{dBSPL}$ e $12=70 \mathrm{dBSPL})$ se evidencia un franco aumento de la presencia de EOApd que varía desde $100 \%$ en $1.500 \mathrm{~Hz}$ hasta $58,6 \%$ en $8.000 \mathrm{~Hz}$ (Tabla 4). Cabe señalar que este comportamiento se mantiene al realizar el análisis por género y oído.

Se pudo evidenciar dos peak notorios que corresponden a las frecuencias de $1,5 \mathrm{kHz}$ y $6 \mathrm{kHz}$ (Figura 1). Se observa un comportamiento similar en las tres intensidades de los tonos primarios, siendo el peak más bajo obtenido en $8 \mathrm{kHz}$ (Figura 1). Así mismo se pudo observar una amplitud mayor de EOApd (I1 = $65,12=55 \mathrm{~dB}$ SPL) en mujeres (Figuras 2 y 3). En relación al comportamiento de las EOApd según oído se pudo observar una mayor amplitud en el odio derecho (Figuras 4,5 y 6 ). 
Tabla 4. Presencia de EOApd ( $\mathrm{n}=70$ oídos) para diferentes intensidades de tonos primarios

\begin{tabular}{|lccc|}
\hline Frecuencia & $\begin{array}{c}\text { Intensidad baja } \\
(50-50 \mathrm{dBSPL})\end{array}$ & $\begin{array}{c}\text { Intensidad media } \\
(50-65 \mathrm{dBSPL})\end{array}$ & $\begin{array}{c}\text { Intensidad alta } \\
(70-70 \mathrm{dBSPL})\end{array}$ \\
\hline $1,5 \mathrm{kHz}$ & $45,8 \%$ & $100 \%$ & $100 \%$ \\
$2 \mathrm{kHz}$ & $55,8 \%$ & $88,6 \%$ & $98,6 \%$ \\
$3 \mathrm{kHz}$ & $45,8 \%$ & $95,8 \%$ & $100 \%$ \\
$4 \mathrm{kHz}$ & $51,5 \%$ & $92,8 \%$ & $100 \%$ \\
$6 \mathrm{kHz}$ & $50 \%$ & $91,5 \%$ & $98,6 \%$ \\
$8 \mathrm{kHz}$ & $1,5 \%$ & $37,2 \%$ & $58,6 \%$ \\
\hline
\end{tabular}

${ }^{*}$ Presencia EOApd (pasa) = 6/6, SNR $+6 \mathrm{~dB}$.

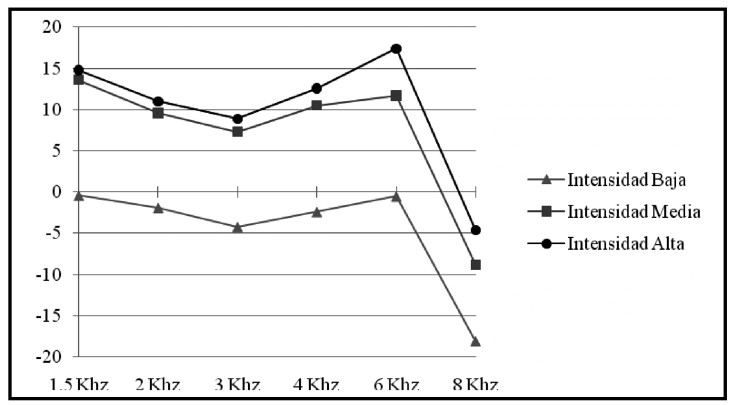

Figura 2. Amplitud promedio ( $\mathrm{dB} \mathrm{SPL}$ ) para género femenino en las tres intensidades evaluadas.

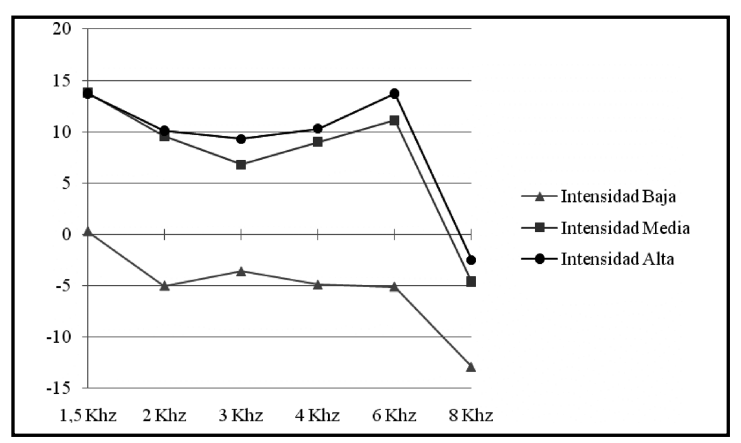

Figura 3. Amplitud promedio (dB SPL) para género masculino en las tres intensidades evaluadas.

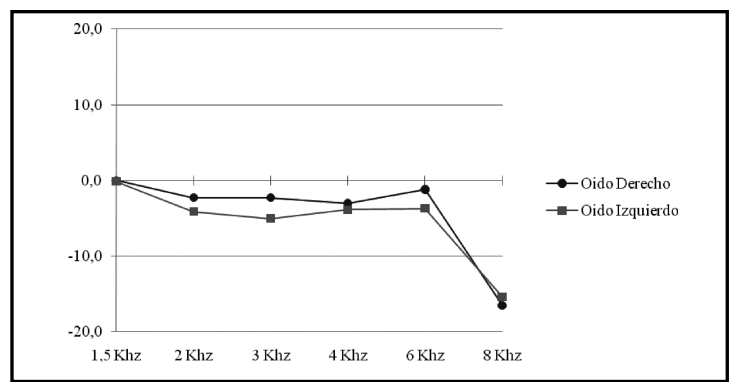

Figura 4. Amplitud promedio (dB SPL) oído derecho e izquierdo para intensidad baja ( $11=50 \mathrm{dBSPL}$ e $12=50 \mathrm{dBSPL})$.
En relación a los umbrales audiométricos se observó un promedio de tonos puros $(500$, 1.000 y $2.000 \mathrm{~Hz})$ de $13,8 \mathrm{~dB}(0,9 \mathrm{DE})$ y de 13 $\mathrm{dB}(1,5 \mathrm{DE})$ para los hombres y mujeres respectivamente.

\section{DISCUSIÓN}

En los resultados obtenidos se evidenció la existencia de una diferencia significativa en la amplitud de las EOApd al variar la intensidad de los tonos primarios. Esto se asemeja a lo observado en estudios similares en donde se variaron los parámetros de intensidad de los tonos primarios encontrándose de igual modo estas diferencias. Es así como según datos recolectados por otros autores, se observó un promedio de amplitud mayor de las EOApd al utilizar intensidades altas de estimulación (I1 $=70 \mathrm{dBSPL}$ e $\mathrm{I} 2=70 \mathrm{dBSPL})^{4}$.

Diversos reportes han evaluado el efecto de los cambios en el nivel de los tonos primarios sobre las EOApd, es así como por ejemplo en uno de ellos se pudo observar para una muestra de 210 individuos que presentaban audición normal o pérdida auditiva ${ }^{3}$ que al analizar los datos en forma separada algunos oídos que presentaban audición dentro de rangos de normalidad no evidenciaron respuesta frente a estímulos de baja intensidad (falsos positivos), es decir, se encontraron sujetos con audición normal con respuesta en intensidades medias y altas, pero sin respuesta a baja intensidad, lo que es semejante a lo observado en nuestra investigación. El mismo 


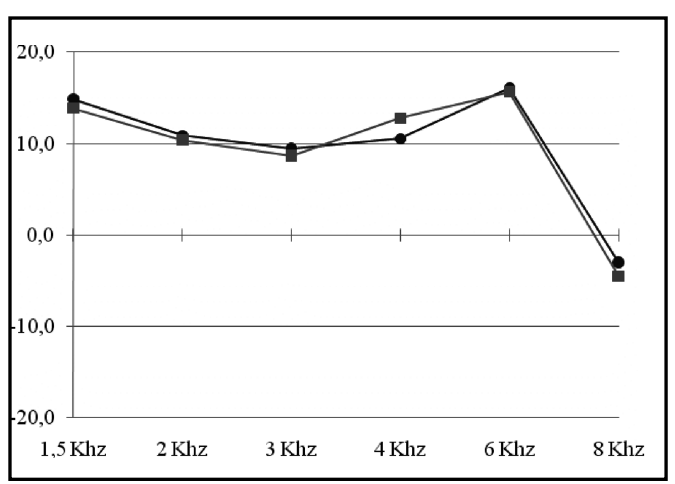

Figura 5. Amplitud promedio (dB SPL) oído derecho e izquierdo para intensidad media $(I 1=55 \mathrm{dBSPL}$ e $\mathrm{I} 2=65 \mathrm{dBSPL})$.

estudio mencionado anteriormente, concluye que a medida que la intensidad de los tonos primarios aumenta, la presencia de EOA también lo hace, por lo tanto disminuye la probabilidad de observar de falsos positivos al menos en sujetos que presentan audición normal. Si bien es cierto el criterio de presencia y ausencia utilizado fue bastante estricto (6/6), el comportamiento observado fue similar a lo evidenciado en la literatura ${ }^{3,4}$.

Diversos estudios atribuyen las diferencias de respuesta en la amplitud frente a los cambios de tonos primarios a mecanismos lineales y no lineales de la cóclea, en donde se evidencia que con pocas excepciones la mayor respuesta de EOApd en sujetos con audición normal se observa en respuesta a estímulos de alta intensidad ${ }^{4}$. Algunos reportes han demostrado que la no linealidad coclear genera productos de distorsión de mayor amplitud a un mayor nivel de estímulo y productos de distorsión de menor amplitud a nivel de estímulos bajos ${ }^{5}$. Esto sugiere la hipótesis de que esto es causado por un fenómeno de ganancia y amortiguación en ambos sentidos entre la sonda ubicada en el CAE y un punto de la membrana basilar ${ }^{5}$. Así mismo se ha propuesto un modelo en el cual la generación de EOApd se producen por la suma de dos componentes: Un mecanismo activo y un mecanismo pasivo. El mecanismo pasivo es virtualmente ausente a bajos niveles de estimulación, pero crece rápidamente cuando el estímulo es más alto.

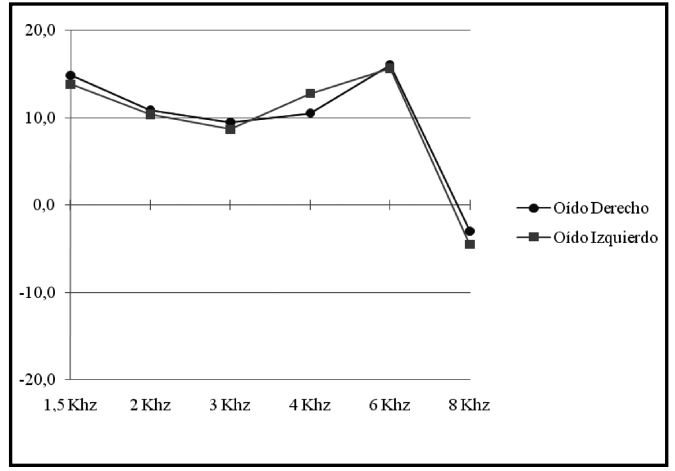

Figura 6. Amplitud promedio (dB SPL) oído derecho e izquierdo para intensidad alta ( $11=70 \mathrm{dBSPL}$ e $12=70 \mathrm{dBSPL})$.

Así mismo, estudios sugieren la existencia de una baja respuesta (amplitud) en las frecuencias bajas en las EOApd. A esto se le puede atribuir algunos factores como existencia de diferencias en la cantidad de distorsión no lineal que se genera en el ápex de la cóclea en comparación con la base de ésta. También puede existir una diferencia frecuencial en la transmisión de energía hacia y de vuelta en el oído medio4. La reducción de la respuesta en esta frecuencia puede corresponder a que tiende a existir un gran sistema de distorsión para $8 \mathrm{kHz}$ en comparación con las frecuencias bajas. Evidencia reciente sugiere que las condiciones de estímulo comúnmente usadas pueden no ser las óptimas para generar EOApd en $8 \mathrm{kHz}^{6,7}$.

Con respecto a las diferencia de amplitud entre género y oído, se pudo observar una similitud a lo observado por Pavlovcinová (2009), en donde no existe un efecto significativo de género en ninguno de estos parámetros (intensidad) de la amplitud de las EOApd. Resultados similares se evidencian en otros estudios recientes que revelan que las diferencias por género de las EOApd son generalmente pequeñas, por lo cual no resultan ser estadísticamente significativas ${ }^{8}$. Por lo tanto no tendría una significancia desde el punto de vista clínico.

Se ha descrito en diversas investigaciones que mujeres presentan promedios $(11=65, I 2=55 \mathrm{~dB}$ $\mathrm{SPL}$ ) de amplitudes de OEApd mayores que los promedios de amplitudes de hombres pero sin ser éstos significativos ${ }^{4}$, lo cual es replicado en los resultados obtenidos en el presente estudio en 
relación a la amplitud de las EAOpd. Esto podría ser debido a que generalmente las mujeres poseen una mayor sensibilidad auditiva que los hombres, sumado a que se cree que existirían diferencias anatómicas y fisiológicas a nivel coclear entre géneros ${ }^{4}$. En nuestro trabajo se observó una leve mejor sensibilidad auditiva en las mujeres.

Con respecto a la variable de oído, se puede concluir que no se observan diferencias significativas en las amplitudes entre ambos oídos, lo cual es similar a lo encontrado por Pavlovcinová, quien determinó que estas diferencias eran pequeñas y no estadísticamente significativas, como sí lo son en las EOA transientes. Por otro lado diversos autores han descrito mayores amplitudes en las EOA de los oídos derechos, que son resultados similares a los obtenidos en el presente trabajo al estimular con baja intensidad, siendo menos evidente esta diferencia para las intensidades medias y altas, sin embargo esta diferencia es pequeña y nuevamente no es estadísticamente significativa. Keogh y cols, demostró una asimetría de oídos prominente en frecuencias altas. Una explicación para esta asimetría sería la generación de una asimetría prenatal en el sistema auditivo durante el primer trimestre del embarazo. Se propone que el género y la asimetría de oído son resultado de diferencias en las fuerzas de inhibición del sistema eferente en la cóclea. La cantidad de inhibición eferente es relativamente menor en el oído derecho y en mujeres, que en los oídos izquierdos y en hombres. Es posible que la diferencia entre oídos en el sistema eferente se deba a asimetrías corticales bien conocidas que definen la producción del lenguaje, su percepción y otras habilidades humanas ${ }^{9}$.

Sería de interés continuar con la línea de investigación y conocer el efecto de los cambios en la intensidad de los tonos primarios en individuos que presentan una hipoacusia de modo de poder establecer si estos efectos son replicados en esa población.

\section{CONCLUSIÓN}

En la presente investigación fue posible evidenciar que la variación de la intensidad de los tonos primarios tiene un efecto estadísticamente significativo sobre las amplitudes de las EOApd en sujetos con audición normal, es así como:

- Intensidades bajas $(I 1=50 \mathrm{dBSPL}$ e 12 $=50 \mathrm{dBSPL}$ ) producen una baja amplitud en sujetos con audición normal junto con una baja presencia de EOApd en las frecuencias de 1.500 $\mathrm{Hz}$ a $8.000 \mathrm{~Hz}$.

- Intensidades medias y altas ( $11=55 \mathrm{dBSPL}$ e 12 $=65 \mathrm{dBSPL}$ y $11=70 \mathrm{dBSPL}$ e $12=70 \mathrm{dBSPL}$ respectivamente) producen una amplitud mayor en sujetos con audición normal junto con un aumento en la presencia de EOApd en frecuencias de $1.500 \mathrm{~Hz}$ a $8.000 \mathrm{~Hz}$.

- En las 3 intensidades estudiadas (bajas, medias, altas) se pudo observar que la frecuencia de $8.000 \mathrm{~Hz}$ es la que presenta la menor amplitud así como una menor presencia de EOApd.

Así mismo se puede concluir que no hay diferencias estadísticamente significativas de amplitud de OEApd entre género y oídos al variar los tonos primarios a distintas intensidades en sujetos con audición normal, de manera que estas variables no inciden en la evaluación y utilidad clínica de las OEApd.

Por lo anteriormente expuesto se puede señalar que la utilización de tonos primarios de baja intensidad para la generación de EOApd no sería adecuada para una correcta identificación del estado de la función coclear al menos en población de sujetos con audición dentro de rangos de normalidad. Debido a esto es importante considerar este factor al momento de variar la intensidad de los tonos primarios en la estimulación para obtener EOApd.

\section{Agradecimientos}

T.M. Fabiola Aránguiz. División de Investigación y Docencia, Signalmed Ltda.

\section{BIBLIOGRAFÍA}

1. Horn J, Pratt SR. Durrant JD. Parameters to Maximize 2f2-f1 Distortion Product Otoacoustic Emission Levels. Journal of Speech, Language, and Hearing Research 2008; Vol. 51: 1620-29. 
2. Katz J. Handbook of clinical audiology. En: Otoacustics Emissions. $5^{\circ}$ edición: Williams \& Wilkins, 2005; 442-51.

3. Stover L, Gorga MP, Neely ST, Montoya D. Toward optimizing the clinical utility of distortion product otoacoustic emission measurements. J Acoust Soc Am 1996; 100(2 Pt 1): 956-67.

4. M RobinetTe \& T GlatTKe. Otoacoustic emissions: clinical applications. En: Distortion-Product Otoacoustic Emissions in relation to hearing loss. 3 Edición: Thieme, 1997; 211.

5. Cheng J. Signal processing approaches on otoacoustic emissions. Unit of Technical and Clinical Audiology, Unit of Technical and Clinical Audiology, Karolinska Institutet, Stockholm, Sweden. 2000; 29-30.
6. Johnson TA, Neely ST, Kopun JG, Gorga MP. Reducing reflected contributions to ear-canal distortion product otoacoustic emissions in humans. J Acoust Soc Am 2006; 119(6): 3896-907.

7. Neely ST, Johnson TA, Gorga MP. Distortionproduct otoacoustic emission measured with continuously varying stimulus level. J Acoust Soc Am 2005; 117(3 Pt 1): 1248-59.

8. SuÁrez H, Velluti RA. La cóclea fisiología y patología. En: Estudio funcional de la audición. $1^{\circ}$ edición: Trilce, 2001; 112.

9. Pavlovcinová G. A normative study of otoacustic emissions, ear asymmetry, and gender effect in healthy schoolchildren in Slovakia. Int J Pediatr Otorhinolaryngol2010; 74(2): 173-7. Epub 2009 Dec 16. 